\title{
Todorov Nicola-Peter, L'administration du royaume de Westphalie de 1807 à 1813, le département de l'Elbe
}

Sarrebruck, éditions universitaires européennes, 2011, 632 p.

Philippe Boulanger

\section{CpenEdition}

\section{Journals}

Édition électronique

URL : https://journals.openedition.org/geohist/1362

DOI : 10.4000/geohist.1362

ISSN : 2264-2617

Éditeur

Association française de la Revue de géographie historique

Référence électronique

Philippe Boulanger, «Todorov Nicola-Peter, L'administration du royaume de Westphalie de 1807 à 1813, le département de l'Elbe », Revue de géographie historique [En ligne], Comptes-rendus, mis en ligne le 20 novembre 2015, consulté le 12 juin 2021. URL : http://journals.openedition.org/geohist/1362 ; DOI : https://doi.org/10.4000/geohist.1362

Ce document a été généré automatiquement le 12 juin 2021.

\section{(i) $\$$}

Ce(tte) œuvre est mise à disposition selon les termes de la Licence Creative Commons Attribution Pas d'Utilisation Commerciale - Pas de Modification 4.0 International. 


\section{Todorov Nicola-}

\section{Peter, L'administration du royaume de} Westphalie de 1807 à 1813, le département de l'Elbe

Sarrebruck, éditions universitaires européennes, 2011, 632 p.

\section{Philippe Boulanger}

\section{RÉFÉRENCE}

Todorov Nicola-Peter, L'administration du royaume de Westphalie de 1807 à 1813, le

département de l'Elbe, Sarrebruck, éditions universitaires européennes, 2011, 632 p.

1 Nicola-Peter Todorov nous livre une remarquable étude sur un sujet à la fois historique et de géographie historique. Dans le contexte actuel de commémoration de la fin de l'Empire napoléonien (la bataille de Waterloo de 1815), son ouvrage participe à mieux connaître l'apport des réformes napoléoniennes, issues du Consulat et de l'Empire, dans la nouvelle structure territoriale et politique en Europe du Nord qu'est le royaume de Westphalie. Il faut dire que l'auteur, docteur en histoire de l'université PanthéonSorbonne, aussi enseignant à l'Université de Rouen et au lycée Gustave Flaubert (académie de Normandie), dispose de nombreuses qualités pour nous exposer une étude scientifique de grande qualité qui donne à voir une autre manière de considérer l'administration de l'Empire napoléonien. Son étude est le fruit de quinze années de travail dans les centres d'archives de Berlin et de Magdebourg, de Paris et de SaintPétersbourg que la maîtrise du français, du russe, de l'allemand et de l'anglais a rendu possible. Le résultat donne lieu à un ouvrage précis, démonstratif, illustré de nombreuses cartes inédites (58 au total), lui conférant ainsi une approche en géographie historique originale, dans un style clair et compréhensible. Il apparaît 
surtout innovant par le résultat produit qui va à contre-courant de la thèse retenue par l'historiographie nationaliste ancienne et, en partie, plus récente.

2 Après la paix de Tilsit de 1807, qui conduit au contrôle des anciennes provinces prussiennes par les armées de Napoléon et à leur rattachement au nouveau royaume de Westphalie, sous la direction de Jérôme Bonaparte, une série de réformes administratives et sociales est menée. L'historiographie donne une vision assez pessimiste de l'application de ces réformes. Celles-ci auraient été mal acceptées, mal appliquées et, surtout, rejetées aussi bien par les populations que par les élites. NicolaPeter Todorov nous livre une autre vision beaucoup moins négative et relativise beaucoup les thèses précédentes. En réunissant des territoires très différents (appartenant au roi de Prusse, à l'électeur de Hesse-Cassel, à l'électeur de Hanovre et au duc de Brunswick), les serviteurs de Napoléon parviennent à créer un royaume de Westphalie qui est sensé devenir un Etat modèle selon les principes politiques et sociaux français en Europe du Nord. Les réformes mises en œuvre sont profondes: application d'une constitution, adaptée de celle de la France impériale, réalisation des huit départements supposant une nouvelle répartition territoriale, adoption du code civil, disparition de l'ordre seigneurial et création de la citoyenneté (impliquant la liberté individuelle et religieuse, l'égalité et la disparition du statut de serf), nouvelle organisation économique. A partir de l'étude du département de l'Elbe en particulier, l'auteur nous donne à comprendre leur mise en œuvre à différentes échelles spatiales (la commune, le district, le département, le royaume) par la consultation d'un corpus de sources diversifiées, reposant aussi bien sur les rapports de l'inspection forestière et les correspondances des préfets que sur les pétitions paysannes. La richesse de cet ouvrage est liée ainsi à son angle d'approche, valorisant le lien entre les hommes et leurs territoires, l'enracinement social des serviteurs de l'Etat et leurs relations avec la société globale.

3 Quel est le résultat de la politique napoléonienne menée dans le royaume de Westphalie? Pour répondre à cette question, l'auteur articule sa démonstration en dix chapitres. Le premier porte sur les relations franco-prussiennes de 1803 à la création du royaume de Westphalie dont la création constitue un enjeu géopolitique en Europe du Nord. Le deuxième aborde l'organisation du nouvel Etat à différentes échelles géographiques, notamment en insistant sur les spécificités locales, tandis que le troisième analyse les pratiques administratives des fonctionnaires français et westphaliens à l'échelle préfectorale et sous-préfectorale. Dans les trois chapitres suivants, ce sont différents secteurs d'activités du Royaume qui permettent de saisir les nuances de l'application des réformes en matière de justice, de finances et progrès social, de la gestion des biens de l'Etat et des forêts, de l'administration des postes et de l'armée. Enfin, dans le dixième et dernier chapitre, l'auteur s'intéresse à la participation de la population dans les ambitions réformatrices françaises et à leur représentation au sein de l'opinion, dont les aspects négatifs (exactions des soldats français, rixes entre civils et soldats ivres par exemple) ont été entretenus dans les manuels scolaires allemands dans les décennies suivantes.

4 Finalement, Nicola-Peter Todorov nous amène à comprendre toutes les nuances de l'ensemble des réformes qui sont appliquées en tenant compte des contraintes locales, parfois à des rythmes différents selon les secteurs d'activités, tout en ayant pour objectif de briser les résistances des élites anciennes. "La division territoriale, surtout cantonale, illustre cette idée à merveille » écrit l'auteur, car les nouvelles entités créées 
permettent de détruire les anciens territoires seigneuriaux, brasser les populations, construire une nouvelle hiérarchie et réaliser de nouvelles dynamiques économiques. L'auteur met ainsi en évidence que la réforme napoléonienne et les agents au service du nouveau royaume, enclins à une "profonde compréhension des hommes » et évitant une rationalité formelle, ont réussi là où les anciens administrateurs prussiens avaient échoué. Les réformes françaises sont ainsi adaptées en fonction de ceux qui les mettent en œuvre et selon les conditions locales. La structure communale continue ainsi d'exister bien après la chute de l'Empire (jusqu'en 1833). Les structures créées par l'Etat français, notamment dans le domaine de la justice, disparaissent ensuite mais ont servi de modèle à la réorganisation ultérieure demandée par le roi de Prusse. En revanche, les réformes sociales ont profité durablement à toute la société au détriment des élites anciennes qui perdent leur emprise sur l'appareil d'Etat. Finalement, l'apport de la période napoléonienne, surtout rendu négatif par l'historiographie nationaliste ancienne, se révèle bien plus profond et à nuancer selon les couches sociales et les secteurs d'activités. Toute la démonstration de l'ouvrage, constituant une somme de connaissances inédites, tend à en révéler toutes les spécificités. 